University of Nebraska - Lincoln

DigitalCommons@University of Nebraska - Lincoln

Faculty Publications, Department of Psychology

Psychology, Department of

2008

\title{
A Review of the Tripartite Model for Understanding the Link between Anxiety and Depression in Youth
}

\author{
Emily R. Anderson \\ University of Nebraska-Lincoln \\ Debra A. Hope \\ University of Nebraska-Lincoln, dhope1@unl.edu
}

Follow this and additional works at: https://digitalcommons.unl.edu/psychfacpub

Part of the Psychiatry and Psychology Commons

Anderson, Emily R. and Hope, Debra A., "A Review of the Tripartite Model for Understanding the Link between Anxiety and Depression in Youth" (2008). Faculty Publications, Department of Psychology. 591. https://digitalcommons.unl.edu/psychfacpub/591

This Article is brought to you for free and open access by the Psychology, Department of at DigitalCommons@University of Nebraska - Lincoln. It has been accepted for inclusion in Faculty Publications, Department of Psychology by an authorized administrator of DigitalCommons@University of Nebraska - Lincoln. 

permission.

\title{
A Review of the Tripartite Model for Understanding the Link between Anxiety and Depression in Youth
}

\author{
Emily R. Anderson, Debra A. Hope \\ University of Nebraska-Lincoln, Department of Psychology, Lincoln, Nebraska, USA \\ anderso3@bigred.unl.edu, dhope1@unl.edu
}

Version of record received September 16, 2006; received in revised form April 21, 2007; accepted May 18, 2007

\begin{abstract}
Although research from numerous investigations indicates that there is substantial overlap in anxiety and depressive symptoms and comorbid diagnoses in youth, these constructs can be adequately differentiated. Clark and Watson [Clark, L. A. \& Watson, D., (1991). Tripartite model of anxiety and depression: Psychometric evidence and taxonomic implications. Journal of Abnormal Psychology, 100: 316-336] proposed a tripartite model to account for the symptom overlap and diagnostic comorbidity between anxiety and depression. This tripartite model posits that anxiety and depression share a common component of negative affect, but can be differentiated by low positive affect associated with depression and high physiological hyperarousal associated with anxiety. The present article reviews initial research which has supported the utility of the tripartite model for explaining the association between anxiety and depression in adult and youth samples. Following that review, more recent investigations which have called into question the applicability of the tripartite constructs for youth are presented. Finally, the paper reviews evidence suggesting that the tripartite factors may not function similarly across all anxiety and depressive disorders. This article concludes by suggesting that more research is necessary with children and adolescents in order to determine the functioning of tripartite constructs across anxiety disorders in youth.
\end{abstract}

Keywords: Tripartite model; Anxiety; Depression; Social anxiety; Adolescence

Contents

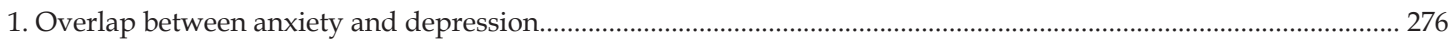

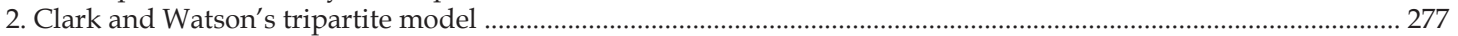





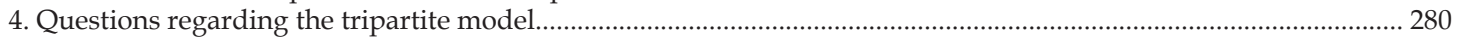

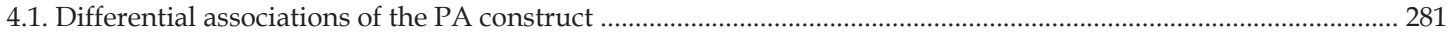

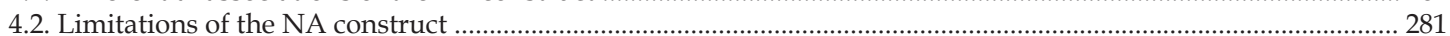



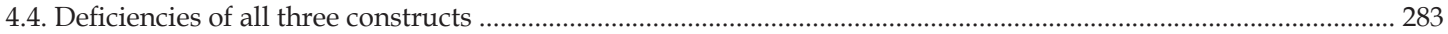

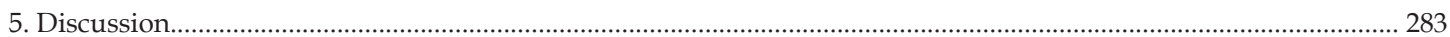

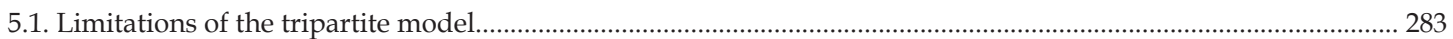

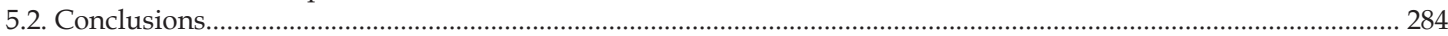

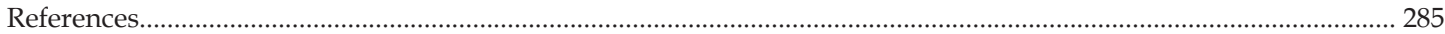

Extensive research indicates that symptoms of anxiety and depression overlap in youth, as evidenced by high intercorrelations between self-report measures of anxiety and depression (e.g., Stark and Laurent, 2001) and by high rates of comorbidity between anxiety and depressive disorders (e.g., Brady and Kendall, 1992). Clark and Watson (1991) proposed a tripartite model to account for the observed overlap between anxiety and depressive symptoms, as well as to explain high comorbidity rates between the two diagnostic categories. The utility of this model in explaining symptom overlap and comorbidity of anxiety and depression has been evaluated and supported in both adults and youth. Some research, however, questions the utility of the tripartite model across the broad category of anxiety disorders. Despite the fact that anxiety and depressive symptoms, as well as diagnoses, are prevalent in children and adolescents, far less research has critically evaluated the broad applicability of the tripartite model in this population as compared to research with adults.

The purpose of this paper is to examine the utility of the tripartite model for understanding the link between 
anxiety and depression in youth and to evaluate the applicability of the tripartite model across different anxiety disorders in youth. This review of the literature will highlight the overlap of anxiety and depressive symptoms and high rates of comorbid diagnoses in children and adolescents. Evidence supporting Clark and Watson's (1991) tripartite model as a means of explaining symptom overlap and diagnostic comorbidity will be reviewed in adults and youth. Finally, research questioning the applicability of the tripartite constructs and limitations of the model will be delineated.

\section{Overlap between anxiety and depression}

Research (e.g., Brady and Kendall, 1992; Seligman and Ollendick, 1998) indicates that there is considerable overlap between anxiety and depressive symptoms in youth, as measured by commonly used self-report questionnaires. The high correlations between measures of anxiety and depression suggest that current selfreport measures demonstrate poor discriminant validity (Brady and Kendall, 1992; Valentiner, Gutierrez, and Blacker, 2002). Brady and Kendall (1992) reviewed a number of studies with children and adolescents and found that correlations between self-report measures of anxiety and depression typically range from $r=0.50$ to $r=0.70$. One explanation for these high correlations is overlapping items on the measures that lead to an overestimation of the association between the two constructs.

In order to determine whether or not overlapping items may in fact account for high associations between self-report measures, two investigations with youth excluded all overlapping items from two commonly used self-report measures in youth, the Children's Depression Inventory (CDI; Kovacs, 1992) and the Revised Children's Manifest Anxiety Scale (RCMAS; Reynolds \& Richmond, 1978). Stark and Laurent (2001) found that after excluding a majority of items from both self-report measures, the correlation between the total scores on these abbreviated measures was $r=0.34$. Cole, Truglio and Peeke (1997) found that analyses using the modified versions of the selfreport measures only reduced the shared variance in trait constructs by $12 \%$ to $14 \%$. In this study, before deleting the overlapping items, the shared variance between anxiety and depression factors was between $52 \%$ and $72 \%$, depending on the type of analysis conducted. Results from these two investigations support Seligman and Ollendick's (1998) assertion that item overlap alone does not fully account for the association between anxiety and depressive symptoms in children, and suggest the presence of at least some degree of shared variance.

Some researchers recognize that the constructs of anxiety and depression are highly correlated, yet suggest that the constructs can be differentiated in a variety of youth samples. Epkins and Meyers (1994) and Crowley and Emerson (1996), using the CDI and RCMAS, found strong associations between anxiety and depression in school children; however, results indicated that a two-factor model, with separate factors of anxiety and depression, best fit their samples. In a population of psychiatric inpatient children, Stavrakaki, Vargo, Boodoosingh, and Roberts (1987) also found support for the differentiation of anxiety and depressive symptoms using the CDI and RCMAS, which suggests that even among children with more severe symptomatology, anxiety and depressive symptoms can be differentiated. Boyd and Gullone (1997) found that anxiety and depression were significantly correlated with each other among nonreferred adolescents using the RCMAS and a different self-report measure of depression, the Reynolds Adolescent Depression Scale (RADS; Reynolds, 1986; $r=0.69$ ). However, similar to the previous investigations, exploratory factor analyses revealed that anxiety and depression loaded on separate and distinct factors.

In addition to the substantial correlations between anxiety and depressive symptoms, there is also considerable comorbidity of anxiety and depressive diagnoses. In a review of the literature, Brady and Kendall (1992) found a $16 \%$ comorbidity rate for anxiety and depressive disorders in community, non-treatment-seeking youth samples. Comorbid anxiety in youth with primary depressive disorders tends to be more common than comorbid depression in youth with primary anxiety disorders (Merikangas \& Avenevoli, 2002). Rates of anxiety comorbidity in depressive youth range from $20 \%$ to $75 \%$ in children and adolescent community samples, whereas rates of comorbid depressive disorders in children and adolescents with anxiety disorders range from $5 \%$ to $55 \%$ in the same samples.

Rates of comorbidity of anxiety and depressive diagnoses seem to be increased in clinical samples of youth, or treatment-seeking youth, with comorbidity rates ranging from $28 \%$ to $62 \%$ (Brady \& Kendall, 1992). Similar to community samples, rates of comorbid anxiety in depressive youth are higher, ranging from $25 \%$ to $50 \%$ (Angold \& Costello, 1993; Axelson \& Birmaher, 2001), than rates of comorbid depression in anxious youth, ranging from $10 \%$ to 15\% (Axelson \& Birmaher, 2001). Angold and Costello (1993) found much higher rates of comorbid depression in anxious youth, however, ranging from $30 \%$ to $75 \%$, which suggests that rates in treatment-seeking youth may be more variable compared to those in community samples. Research consistently indicates that in general, depressed individuals are likely also to be anxious, but those youth who are anxious are less likely also 
to be depressed (Finch, Lipovsky, \& Casat, 1989; King, Ollendick, \& Gullone, 1991; Ollendick, Shortt, \& Sander, 2005). This research has lead to the proposition that there may be a temporal relationship between anxiety and depression such that anxiety disorders may lead to concurrent depression (Brady \& Kendall, 1992).

There is in fact substantial evidence that anxiety disorders tend to precede the onset of depressive disorders (Bittner et al., 2004; Brady \& Kendall, 1992; Chorpita \& Daleiden, 2002; King et al., 1991; Kovacs, Gastonis, Paulauskas, \& Richards, 1989; Merikangas \& Avenevoli, 2002), and may increase the risk of subsequent depression (Bittner et al., 2004; Cole, Peeke, Martin, Truglio, \& Seroczynski, 1998). Orvaschel, Lewinsohn and Seeley (1995) found that $66 \%$ of adolescents with a primary diagnosis of an anxiety disorder later developed a second diagnosis of depression, whereas only $6.5 \%$ of adolescents who first had depression later developed an anxiety disorder. Thus, youth with anxiety disorders may subsequently develop symptoms of depression, or even depressive diagnoses, associated with their unremitting anxiety symptoms.

In addition to support for a temporal association between anxiety and depression, certain characteristics consistently have been found in youth with comorbid anxiety and depression, at both the symptom and diagnostic levels. First, children with both anxiety and depressive symptoms tend to be older than their anxious only or depressed only counterparts (Brady \& Kendall, 1992; Merikangas \& Avenevoli, 2002; Strauss, Last, Hersen, \& Kazdin, 1988). In fact, the highest comorbidity rates of anxiety and depressive disorders have been found among adolescents (Ollendick et al., 2005). Second, highly anxious adolescents have been shown to present clinically similarly to depressed adolescents (King et al., 1991). Specifically, adolescents with severe anxiety were found to report more depressive symptoms such as dysphoria, low self-esteem, anhedonia, and suicidal ideation compared to adolescents with lower anxiety, who report less depressive symptomatology.

\section{Clark and Watson's tripartite model}

One important finding gleaned from the evidence reviewed thus far is that anxiety and depression share an underlying similarity, but also are comprised of unique characteristics. Clark and Watson (1991) proposed a tripartite model to best capture the existing data on the extensive overlap of anxiety and depressive symptoms and comorbidity. This model initially was proposed to account for overlap and comorbidity in adults, but it also has been applied to children and adolescents. The model posits that anxiety and depression share a common component of negative affect (NA) which accounts for symptom overlap and comorbidity. Negative affect (NA) represents the extent to which an individual feels upset or unpleasantly engaged, rather than peaceful. NA also indicates a sense of high objective distress and encompasses a variety of affective states including upset, angry, guilty, afraid, sad, scornful, disgusted, and worried. Clark and Watson suggested that the states of calm and relaxed represent a lack of NA, and that commonly used self-report measures of anxiety and depression measure this shared trait of NA. As noted earlier, self-report measures of anxiety and depression are highly correlated, and item overlap only accounts for a small amount of the shared variance between these measures, which supports the notion that they tap into the underlying construct of NA.

Clark and Watson hypothesized that although anxiety and depression share a common component of NA, they can be differentiated by two constructs: positive affect (PA) and physiological hyperarousal (PH). Clark and Watson's research found that individuals with symptoms or diagnoses of depression tend to exhibit low levels of PA and high levels of NA, whereas individuals with anxiety disorders tend to exhibit high levels of $\mathrm{PH}$ as well as high levels of NA. Positive affect (PA) represents pleasurable engagement with the environment, and the extent to which a person feels a zest for life, enthusiastic, alert, and active. PA encompasses mood states including energetic, pleasurable engagement, active, delighted, interested, enthusiastic, and proud. Clark and Watson suggested that the absence of PA is reflected by terms such as tired, fatigued, and sluggish. Physiological hyperarousal (PH) includes somatic tension, short of breath, dizziness, lightheadedness, and dry mouth.

Although the construct of PH has not been studied very much until recently, the tripartite constructs of PA and NA have garnered nearly two decades of empirical support (Tellegen, Watson, \& Clark, 1999). Although some research has found that these factors are relatively orthogonal (Choripta, Daleiden, Moffitt, Yim, \& Umemoto, 2000; Laurent, Catanzaro, \& Joiner, 2004), the findings have been mixed. In a study of community adolescents, Huebner and Dew (1995) found that PA and NA were weakly correlated $(r=-0.13)$, as measured by the Positive and Negative Affect Schedule (PANAS; Watson, Clark \& Tellegen, 1988), similar to results from adult studies. A number of studies, however, have reported that PA and NA are more highly correlated (Brown, Chorpita \& Barlow, 1998; Lonigan, Hooe, David, \& Kistner, 1999; Watson, Clark, \& Carey, 1988; Watson, Webber et al., 1995). For example, Crook, Beaver, and Bell (1998) found a significant correlation in schoolchildren between the constructs of NA and PA $(r=-0.39)$, as measured by the Positive and Negative Affect Scale for Children (PANAS-C), which is higher than correlations reported in other studies $(r=-0.12$ to $r=-0.28$; Watson et al., 1988). It is possible 
that PA and NA may be more highly associated in younger children compared to adolescents and adults. However, research is fairly inconsistent with regard to the orthogonality of the PA and NA constructs.

Initial empirical research has sustained the utility of the tripartite model for explaining the association between anxiety and depression in adults. Research with undergraduate students supported considerable overlap of symptoms of anxiety and depression; however, the two constructs were distinguished best using a three-factor solution, including the constructs of PA, NA, and PH (Joiner, 1996; Watson, Clark et al., 1995). In fact, using the PANAS, the most commonly used measure of positive and negative affect, the PA factor significantly loaded on the depression construct, as hypothesized by the model. Watson, Clark et al. (1995) also investigated the tripartite model in nonclinical adults and patients participating in treatment for substance abuse. Results suggested that differentiation of symptoms of anxiety and depression was improved by deemphasizing the importance of nonspecific symptoms, such as NA, and by focusing instead on symptoms that are relatively unique to each construct, including PA and PH. These initial studies of the tripartite model in diverse adult samples attest to its utility in accounting for symptom overlap and differentiation.

\subsection{Support in clinical and community samples of youth}

Similar to studies with adults, research with youth consistently has supported the utility of the tripartite model in explaining the association between overlapping symptoms and diagnoses of depression and anxiety. Research has supported the utility of the tripartite model in inpatient children and adolescents using the CDI and RCMAS (Lonigan, Carey, \& Finch, 1994). In this investigation, scores on the CDI and RCMAS discriminated between children diagnosed with anxiety and depression, as anticipated by the model. Lonigan and colleagues suggested that the CDI and RCMAS seem to primarily measure NA, but portions of each scale may be more closely related to anxious and depressed symptomatology. Measures related to low PA in this study best discriminated children with depressive disorders from those with anxiety disorders. Joiner, Catanzaro and Laurent (1996) found support for a three-factor model using the PANAS-C and specific items from the CDI and RCMAS, selected on rational grounds by the researchers, to best capture the constructs of PA and NA. Joiner and Lonigan (2000) examined the tripartite model in another sample of children and adolescent psychiatric inpatients using the PANAS-C and the RCMAS and found that combinations of high NA and low PA serve as a risk factor for development and/or maintenance of depressive, but not anxious symptoms. Furthermore, changes over time in symptoms of depression, but not anxiety, were associated with the combination of low PA and high NA. Lastly, results supported a differentiation of diagnostic status based on NA and PA such that these constructs were associated with depressive versus externalizing diagnoses.

Using the CDI and RCMAS to measure PA and NA, several investigations have found support for two-factor models of PA and NA as distinct constructs in school children (e.g., Stark and Laurent, 2001). Results from several studies suggest that anxiety and depression may represent a unified construct in young children that separates into distinct constructs of anxiety and depression with increasing age. Cole et al. (1997) found support for the differentiation of PA and NA among sixth grade students, but not among third grade students. Another investigation by Lonigan et al. (1999) found that in regression models, both PA and NA made significant, unique contributions to the prediction of CDI and RCMAS scores in adolescents, but not in children.

Other investigations have used the PANAS-C or PANAS to measure PA and NA and found support for twofactor models in adolescents (e.g., Huebner \& Dew, 1995) and children. Crook et al. (1998) examined anxiety and depressive symptoms in third through fifth grade students utilizing the CDI, RCMAS, and additionally the PANAS-C and found that the constructs of PA and NA were related to scores on self-report measures of depression and anxiety as predicted by the model, regardless of age. It is possible that these results differ from those of Cole and colleagues and Lonigan and colleagues because the latter investigations included the PANAS-C and PANAS, respectively, which are better measures of PA and NA than total scores on the CDI and RCMAS. Use of the CDI and RCMAS in isolation likely measures NA more so than PA, and thus may not differentiate the two constructs. Taken together, these studies suggest that the tripartite model is useful in explaining the association of anxiety and depressive symptoms in community samples of youth.

In an attempt to avoid using questionnaires that measure solely NA, such as the CDI and RCMAS, Valentiner et al. (2002) utilized several different self-report measures in a nonclinical adolescent sample including the Multidimensional Anxiety Scale for Children (MASC; March, Parker, Sullivan, Stallings, \& Conners, 1997), Spence Children's Anxiety Scale (SCAS; Spence, 1998), and Reynolds Adolescent Depression Scale (RADS). The MASC and SCAS have been purported to be more "pure" measures of anxiety (March et al., 1997), rather than merely measures of NA, and thus should show better discrimination from measures of depression. Results from this investigation indicated that the anxiety measures showed relatively strong, positive relationships with NA and 
small, negligible relationships with PA, in accordance with the tripartite model. Results indicated, however, that the anxiety measures demonstrated relatively poor discriminant validity from the RADS, similar to the findings with more commonly used measures such as the RCMAS and the CDI. Although these newer self-report measures show promise with regard to discriminating PA and NA more effectively than the RCMAS and CDI, which seem to measure only NA, more research is warranted due to the poor discriminant validity found in this study.

Research has not only supported the utility of two-factor models of PA and NA in nonclinical youth samples, but also the utility of the three-factor model in nonclinical samples of youth, including a sample of nonclinical African American urban youthingrades six and nine (Lambert, McCreary, Joiner, Schmidt, \& Ialongo, 2004). Results from an investigation by Phillips, Lonigan, Driscoll, and Hooe (2002), using the CDI, RCMAS, and PANAS-C, indicated that although the presence of three distinct constructs was supported, parent reports of anxiety and depression in their children were not associated with children's endorsements of NA. Phillips and colleagues suggested that some NA characteristics such as low levels of activity, social withdrawal, and lack of active engagement with the environment are observable, whereas internal NA features are more difficult to observe and thus parents may not be accurate reporters of internal experiences of their children, which are a component of NA. These results suggest that parent ratings may not add to the utility of the tripartite model.

Another investigation of the tripartite factors in children in grades three, six, and nine by Turner and Barrett (2003) measured the constructs PA, NA and PH by extracting items from the CDI and RCMAS, as specified in Chorpita, Plummer, and Moffitt (2000). The utility of a three-factor model of NA, PA, and PH was supported at all three grade levels, suggesting that symptoms of anxiety and depression can be differentiated across all age groups. These results are in accordance with some research (i.e., Crook et al., 1998), but differ from those of Cole et al. (1997), who found differentiation at older, but not younger ages. One reason for these discrepancies may be that Cole and colleagues used total CDI and RCMAS scores, and Turner and Barrett used specific items from each scale deemed to measure symptoms of depression and anxiety specifically, rather than just negative affect. Furthermore, although Crook and colleagues used total scores from the CDI and RCMAS, they also utilized scores from the PANAS-C, which is a better measure of PA and NA.

The preceding paragraphs suggest that discrepancies in the utility of the tripartite factors between age groups may result from measurement differences. Investigations that utilize only total scores on the CDI and RCMAS as measures of PA and NA found differentiation between older and younger children, which may be the result of poor discriminant validity of the PA and NA factors. Investigations that use the PANAS as a measure of PA and NA, however, find no significant differences in the factors across age groups. These results suggest that better measurement of the tripartite factors is necessary. Two investigations tested new measures of tripartite constructs, the Physiological Hyperarousal Scale for Children (PH-C; Laurent et al., 2004) and the Affect and Arousal Scale (AFARS; Chorpita, Daleiden et al., 2000) and found support for distinct constructs of NA, PA, and PH in

Table 1

Research supporting the tripartite factors

Researchers

Cole, Truglio, and Peeke (1997)

Crook et al. (1998)

Huebner and Dew (1995)

Inderbitzen and Hope (1995)

Joiner (1996)

Joiner, Catanzaro, and Laurent (1996)

Population

6th (but not 3rd) grade students

3 rd-5th grade students

Adolescents

Adolescents

Undergraduate students

Inpatient children/adolescents

Lambert et al. (2004)

Lonigan et al. (1999)

Lonigan et al. (1994)

Phillips, Lonigan, Driscoll, and Hooe (2002) Children/adolescent students

Stark and Laurent (2001)

Turner and Barrett (2003)

Valentiner et al. (2002)

Watson, Clark et al. (1995) 6th and 9th grade students

Adolescents

Inpatient children

4 th-7th grade students items from

$3 \mathrm{rd}, 6$ th, and 9 th grade students

Adolescents

Undergraduate students, nonclinical PANAS

adults, substance abuse clients

$\begin{array}{ll}\text { Measures used } & \text { Findings } \\ \text { CDI, RCMAS } & 2 \text { factor model } \\ \text { CDI, RCMAS, PANAS-C } & 2 \text { factor model } \\ \text { PANAS } & 2 \text { factor model } \\ \text { CDI, RCMAS, SASC-R } & 2 \text { factor model } \\ \text { BDI, BAI, PANAS } & 3 \text { factor model } \\ \text { Items from CDI, RCMAS, } & 3 \text { factor model } \\ \quad \text { and total PANAS-C } & \\ \text { BHIF } & 3 \text { factor model } \\ \text { CDI, RCMAS } & 2 \text { factor model } \\ \text { CDI, RCMAS } & 3 \text { factor model } \\ \text { CDI, RCMAS, PANAS-C } & 3 \text { factor model } \\ \text { CDI, RCMAS } & 2 \text { factor model } \\ \text { Items from CDI, RCMAS } & 3 \text { factor model } \\ \text { MASC, SCAS, RADS } & 2 \text { factor model } \\ \text { PANAS } & 3 \text { factor model }\end{array}$

PANAS = Positive and Negative Affect Scale, CDI = Children's Depression Inventory, RCMAS = Revised Children's Manifest Anxiety Scale, MASC = Multidimensional Anxiety Scale for Children, SCAS = Spence Children's Anxiety Scale, RADS = Reynolds Adolescent Depression Scale, SASC-R = Social Anxiety Scale for Children-Revised, BHIF = Baltimore How I Feel, PANAS-C = Positive and Negative Affect Scale for Children. 
nonclinical children and adolescents. It is possible that the newer measures of the tripartite constructs may be better able to differentiate across age groups, but more research is needed. Table 1 depicts the studies reviewed in this section.

\section{An alternative conceptualization of the tripartite model}

The preceding paragraphs have summarized empirical research supporting the utility of the tripartite model for explaining the overlap and comorbidity of anxiety and depression in adults, children, and adolescents in both clinical and community samples. Another line of research has garnered support for a slightly different conceptualization of the tripartite model. Chorpita, Albano and Barlow (1998), drawing heavily from clinical research, have suggested that fear represents the third construct in addition to anxiety and depression, rather than PH. In this model, anxiety was conceptualized as distinct from autonomic arousal, or PH. Chorpita and colleagues posited that anxiety is a pure manifestation of NA, PH represents the discrete emotion of fear, and low PA corresponds with depression. This model stipulates that PH encompasses not only fear, but also panic phenomenology, which may be distinct from other anxiety disorders with regard to PH. Furthermore, in this model, anxiety encompasses tension, apprehension, worry, and general distress, whereas depression corresponds to anhedonia, low PA, and hopelessness.

This alternative model is based on previous research that has found differential correlations between the constructs of fear, anxiety, and depression. A review (Muris, Schmidt, Merckelbach, \& Shouten, 2001) of a number of studies found low correlations between symptoms of fear and depression $(r=0.20)$, moderate correlations between symptoms of fear and anxiety $(r=0.38)$, and high correlations between symptoms of anxiety and depression $(r=0.64)$. In two investigations of fear, anxiety, and depressive constructs, Ollendick and Yule (1990) and Ollendick, Yule, and Ollier (1991) found that depression and anxiety, as measured by the CDI and RCMAS, were more closely related than were depression and fear, as measured by the CDI and the Fear Survey Schedule for Children-Revised (FSSC-R; Ollendick, 1983) in eight to ten-year old school children in England. Children who reported high levels of depression also reported high levels of anxiety and social evaluative fears. NA related to specific social situations may characterize these children more so than a global disposition to experience aversive emotional states, as suggested in earlier research.

Two studies have investigated the utility of Chorpita and colleagues' modified version of the tripartite model. Muris et al. (2001) measured depression, fear, and anxiety using the CDI, the FSSC, and the SCAS in a sample of community adolescents. Results indicated that fear, anxiety, and depression were distinct, yet correlated constructs. In a second investigation Chorpita et al. (1998) theoretically selected items from the CDI, RCMAS, and Child Behavior Checklist to measure NA, PA, and fear and utilized the Anxiety Disorders Interview Schedule for Children, for DSM-IV (ADISC; Silverman \& Albano, 1996) to establish diagnostic status in children presenting for outpatient treatment. Results again indicated that NA, PA, and PH were distinct, but correlated factors. As predicted by the model, anxiety corresponded to NA, depression corresponded to low PA, and fear corresponded to PH. Contrary to the predictions of the model, however, PH was correlated not only with fear and panic, but also with depression severity and separation anxiety. NA, furthermore, was not found to be related to social anxiety or depression, as predicted by the model. Based on these findings, Chorpita and colleagues suggested that one problem with the tripartite model is its failure to articulate possible connections among the general higher-order factors of NA, PA, and $\mathrm{PH}$, and specific diagnoses and recommended that future research investigate the fit of tripartite constructs with different diagnostic categories.

\section{Questions regarding the tripartite model}

As reviewed thus far, a great deal of research in adults and youth has supported the applicability of the original tripartite model and a slightly modified version of the model. Another group of investigations, however, has found partial support for the tripartite model, but has called into question some of its theoretical constructs. Watson, Gamez and Simms (2005), echoing Chorpita et al. (1998), noted that one limitation of the tripartite model is its failure to account for the substantial heterogeneity among the anxiety disorders. While the tripartite theory seems to explain unspecified anxiety symptoms, it does not appear to be applicable across anxiety disorders. The tripartite constructs furthermore may show differential correlations with depressive symptoms and diagnoses. Brown et al. (1998) noted that few studies with children have looked at PA, NA, and PH with regard to specific mood and anxiety disorders, and suggest this as an avenue for future research.

One study has examined a particular anxiety disorder in youth (Inderbitzen \& Hope, 1995). They investigated the utility of the tripartite model in tenth grade students, some who endorsed significant symptoms of social phobia. Results indicated that the relationship between symptoms of depression, as measured by the CDI, and 
unspecified anxiety, as measured by the RCMAS, was stronger than the relationship between symptoms of depression and symptoms of social anxiety, as measured by the Social Anxiety Scale for Children-Revised (SASC-R; LaGreca \& Stone, 1993). In fact, the shared variance between symptoms of depression and symptoms of social anxiety was only $16 \%$, whereas the shared variance between depression and unspecified anxiety was $50 \%$. This study was the first study in youth to demonstrate that specific anxiety symptoms, such as social anxiety, are differentially related to symptoms of depression, compared to unspecified anxiety, which has been the focus of most investigations.

\subsection{Differential associations of the PA construct}

Evidence from a number of adult studies suggests that although results support a three-factor structure consistent with the tripartite model, low PA is associated not only with depressive disorders and symptoms, as predicted by the tripartite model, but also with social phobia symptoms and diagnoses in both inpatient (Watson, Clark, and Carey, 1988; Watson, Clark, and Tellegen, 1988) and outpatient (Brown et al., 1998) samples. The latter investigation, in fact, found that low PA, as measured by the PANAS, was more highly correlated with social phobia diagnoses than with all other anxiety disorders. These findings are consistent with previous research that has found correlations between PA and diverse indices of social behavior, including frequency and satisfaction of contact with friends and relatives, ability to make new friends, involvement in social organizations, and trait measures of extraversion or sociability. Watson and colleagues suggested that the negative correlations between PA and social phobia diagnoses is not surprising, given that social phobia reflects fear and distress in interpersonal situations, similar to the disruption of interpersonal functioning that is related to depression.

From a somewhat different approach, Watson et al. (2005) reviewed a number of investigations on adult temperament, including neuroticism and extraversion, with the assumption that neuroticism corresponds to negative emotionality, or NA, and extraversion corresponds to positive emotionality, or PA. In this review, significant associations were found once again between low PA, or extraversion, and social phobia diagnoses. Specifically, the correlation between PA and social phobia $(r=-0.18)$ was significantly higher than correlations between PA and the remaining anxiety disorders (rsranged from -0.03 to -0.06). This investigation provides additional support for conceptualizing low PA as an important characteristic of social phobia as well as depression, contrary to the hypotheses of the tripartite model. As noted, this association makes sense given the relationship between social behavior and low PA.

Correlations between low PA and social phobia have not been investigated fully in youth. One study by Chorpita, Plummer and Moffitt (2000b) examined the tripartite model in a sample of treatment-seeking preadolescents using selected items from the CDI and RCMAS to measure PA and NA, and the ADIS-C interview to establish diagnoses. In accordance with the tripartite model, two higher-order emotion factors, NA and PA, emerged. Results indicated, however, a significant negative correlation not only between PA and depressive diagnoses, as predicted by the model, but also between PA and social phobia diagnoses, which is consistent with the adult research reviewed above. Another investigation by Jacques and Mash (2004) examined the tripartite factors in a sample of elementary and high school students using the CDI, the State-Trait Anxiety Inventory (STAI; Speilberger, Gorsuch, \& Lushene, 1970) and the two measures specifically measuring tripartite constructs, the PH-C and the AFARS. Results indicated that, contrary to the predictions of the tripartite model, and contrary to the results of Watson et al. (2005), significant negative correlations emerged between PA and unspecified anxiety symptoms. Jacques and Mash suggested that these correlations are not particularly surprising due to the high intercorrelations found between the tripartite constructs, as well as the poor discriminant validity of tripartite constructs in this sample. More research is needed in order to better elucidate the relationship between PA and anxiety in youth and to replicate these results, given that this is the only investigation that has found correlations between unspecified anxiety and PA. The results from these two studies with youth suggest that low PA may show differential correlations with not only social phobia diagnoses, but also with unspecified anxiety symptoms.

\subsection{Limitations of the NA Construct}

Evidence from several investigations questions the utility of the NA construct across the broad category of anxiety disorders. Watson et al. (1988) found that NA was correlated with symptoms of anxiety and depression broadly in a sample of adults, as predicted by the model, but that NA did not add significantly to the prediction of social phobia diagnoses, despite the fact that NA had a significant zero-order correlation with the presence of a social phobia diagnosis. Watson and colleagues posited that this finding reflects the significant correlation between NA and PA in this sample $(r=-0.37)$ and that once PA is partialed out, NA is no longer significantly 
correlated with social phobia diagnoses (partial $r=0.12$ ). This finding suggests that PA may account for observed correlations between NA and social phobia.

In another investigation Watson et al. (2005) hypothesized that theoretically, NA should correlate more strongly with distress-based disorders such as major depressive disorder (MDD) and generalized anxiety disorder (GAD) compared to mood and anxiety disorders with more modest distress components, such as social phobia or panic disorder. This review indicated that, in fact, many anxiety disorders, other than GAD, were associated with a significant, but more limited component of NA. Watson and colleagues posited that individuals with other anxiety disorders may experience marked distress, but this distress is likely concentrated in certain situations, such as social situations in individuals with social phobia, or in temporally discrete episodes, such as those associated with panic disorder. Results, furthermore indicated that NA seems to be more weakly related to disorders characterized primarily by behavioral avoidance, such as specific phobias. Thus, contrary to the tripartite model, Watson and colleagues suggested that NA is more strongly related to GAD than other anxiety disorders.

Only one investigation has examined differential associations of NA across anxiety disorders in youth. Chorpita et al. (2000) found that NA, as measured by selected items from the CDI and RCMAS, was significantly correlated with clinical severity ratings of all DSM-IV mood and anxiety syndromes, as measured by the ADIS-C, in treatment-seeking preadolescents except separation anxiety disorder, social phobia, and depression, contrary to the tripartite model's hypothesis that all anxiety and depressive disorders exhibit a shared component of NA. More research is needed in children and adolescents to better evaluate this finding.

\subsection{Limits of the PH construct}

Far fewer studies have examined the construct of $\mathrm{PH}$, compared to NA and PA. Studies have found that PH is significantly associated with panic disorder and not with the other anxiety disorders in outpatient adults (e.g., Brown et al., 1998), and in children (e.g., Chorpita et al., 1998). In the investigation by Brown et al. (1998), PH, as measured by the BAI, was significantly associated with all anxiety disorder diagnoses on the ADIS; however, after controlling for NA, as measured by the PANAS, PH was related only to panic disorder. These results suggested that NA likely plays a large role in observed correlations between PH and anxiety disorders. Joiner, Steer et al. (1999) attempted to identify items consistent with the construct of PH using the Beck Anxiety Inventory (BAI; Beck, Epstein, Brown, \& Steer, 1988) in three diverse adult samples including air force cadets, undergraduate students, and psychotherapy outpatients. Results indicated that six items from the BAI reliably estimated the construct of PH. In this investigation, PH was more highly correlated with panic disorder compared to all other anxiety disorders. These results support the hypothesis that $\mathrm{PH}$ is a particular characteristic of panic disorder, and may not adequately discriminate anxiety disorders from mood disorders as posited by the tripartite model.

In a sample of treatment-seeking children, Chorpita et al. (1998) found that $\mathrm{PH}$, as measured by items from the CDI and RCMAS, was significantly correlated not only with panic disorder diagnoses, but also with separation anxiety and with depressive diagnoses, as measured by the ADIS-C. Chorpita and colleagues suggested that somatic complaints are known to be elevated among children with separation anxiety and that PH is a central feature of panic, which may explain the significant correlations found between $\mathrm{PH}$ and panic and separation anxiety disorder diagnoses. In another study, Chorpita and Daleiden (2002) investigated the tripartite model in a sample of children and adolescents and found that panic disorder symptoms, as defined by clinical severity ratings based on diagnoses on the ADIS-C, showed the strongest correlation with $\mathrm{PH}$, as measured by the $\mathrm{PH}$ scale of the AFARS. One explanation for the failure of this study to find correlations between $\mathrm{PH}$ and separation anxiety is that the AFARS was designed specifically to measure the construct $\mathrm{PH}$, whereas previous investigation utilized the CDI and RCMAS, which were not designed to measure PH. This research thus supports the hypothesis that $\mathrm{PH}$ is a distinguishing feature of panic disorder and is not broadly characteristic of anxiety disorders.

Several investigations also have found support for a significant relationship between $\mathrm{PH}$ and depression, contrary to the tripartite model. Chorpita and Daleiden (2002) found that the construct with the second strongest correlation with $\mathrm{PH}$, after panic disorder, was depression, as measured by the CDI. An investigation with school children, by Jacques and Mash (2004), also found significant correlations between PH and depressive symptoms, as measured by the CDI. Jacques and Mash suggested that the correlations between PH and depressive symptoms may be related to the somatic features of depression, which combine symptoms of anxiety, depression, and somatization. As noted in the present paper, the CDI measures NA more so than depression, and thus a correlation with the CDI may be merely a correlation between the tripartite constructs of $\mathrm{PH}$ and NA. Results from these two studies suggest that more research is needed to elucidate the relationship between depression and PH in youth and that this research should include newer measures of the tripartite constructs. 


\subsection{Deficiencies of all three constructs}

Overall, evidence has failed to support the utility of the tripartite factors across anxiety and depressive disorders, which possibly reflects high intercorrelations and a lack of discriminant validity of the measurements of the tripartite constructs. Two investigations have suggested that all three tripartite constructs may function differently than predicted by the model. A notable study by Burns and Eidelson (1998) failed to support the tripartite constructs in three adult samples including college students, outpatients seeking treatment for substance abuse, and outpatients seeking treatment for mood disorders, anxiety disorders, or both. In all three samples, the nonspecific depression and nonspecific anxiety scales, the BDI and the Burns Anxiety Inventory, could not be successfully loaded onto a single NA factor, as hypothesized by the tripartite model. Results also indicated that nonspecific anxiety and depressive symptoms, rather than PH and PA, were found to be the most valid indicators of anxiety and depression, which suggests that PA and PH may not adequately differentiate anxiety and depression. This study fails to confirm that measures of PA and PH are distinct from general distress variance, or NA. Burns and Eidelson found that a four factor model fit the data best with anhedonia, nonspecific depression, nonspecific anxiety, and somatic arousal representing the four factors.

A second investigation by Ollendick, Seligman, Goza, Byrd, and Singh (2003) failed to support the utility of the tripartite model in nonclinical children and adolescents using the CDI and RCMAS total scales. In this investigation, a two-factor model provided a better fit with the data compared to the tripartite model. The two factors in the model were highly correlated $(r=0.78)$, however, despite efforts to differentiate them, which suggests the presence of a common factor of negative affectivity. As noted in this review paper, the CDI and RCMAS seem to measure NAwhen the total scales are used, and thus this result is not surprising. Ollendick and colleagues noted also that the lack of support for the tripartite model may be related to the poorly defined PA and $\mathrm{PH}$ constructs in this study. Table 2 depicts the studies reviewed in this section.

\section{Discussion}

\subsection{Limitations of the tripartite model}

As noted in this review of the literature, there are several important limitations of Clark and Watson's tripartite model. One of the biggest limitations is the model's failure to account for the substantial heterogeneity among the anxiety disorders (Brown et al., 1998; Watson, 2005; Watson et al., 2005). Research by Mineka, Watson, and Clark (1998) supports the reviewed findings that the tripartite factors may function differentially across the anxiety dis-

Table 2

Research questioning the tripartite factors

Researchers Population

Brown et al. (1998) Adult outpatients

Burns and Eidelson College students, outpatient substance (1998) abusers, outpatient mood/anxiety adults

Chorpita et al. (1998)

Treatment-seeking children

Chorpita and Daleiden (2002)

Chorpita, Plummer et al. (2000)

Jacques and Mash (2004)

Joiner, Blalock et al. (1999); Air force cadets, undergraduate Joiner, Steer et al. (1999) students, and psychotherapy outpatients Ollendick et al. (2003)

Watson, Gamez, and Simms (2005)

Watson et al. (1988) Nonclinical children/adolescents Nonclinical adults Adult inpatients

\begin{tabular}{|c|c|}
\hline \multirow{3}{*}{$\begin{array}{l}\text { Measures used } \\
\text { ADIS, PANAS, BDI, BAI }\end{array}$} & Findings \\
\hline & Low PA and SP \\
\hline & High PH with PD \\
\hline $\begin{array}{l}\text { BDI, Burns Anxiety Inventory, } \\
\text { SCL-90 }\end{array}$ & $\begin{array}{l}4 \text { factor model: anhedonia, non- } \\
\text { specific anxiety, nonspecific } \\
\text { depression, somatic arousal }\end{array}$ \\
\hline ADIS-C, items from RCMAS, CDI & High $\mathrm{PH}$ with $\mathrm{PD}, \mathrm{SAD}$ \\
\hline $\begin{array}{l}\text { ADIS-C, AFARS, PANAS-C, } \\
\text { CDI, RCMAS }\end{array}$ & High PH with, 2nd with MDD \\
\hline ADIS-C, items from RCMAS, & Low PA and SP \\
\hline PH-C, AFARS, STAI, CDI & $\begin{array}{l}\text { Low PA and unspecified anxiety } \\
\text { High PH and depression }\end{array}$ \\
\hline BAI, ASI & High PH with PD only \\
\hline CDI, RCMAS & $2 \mathrm{fa}$ \\
\hline- & Low PA and SP \\
\hline A scal & Low PA and SP \\
\hline MPC & High NA not with SP \\
\hline
\end{tabular}

$\mathrm{PA}=$ positive affect, $\mathrm{PH}=$ physiological hyperarousal, $\mathrm{NA}=$ negative affect, $\mathrm{SP}=$ social phobia, $\mathrm{PD}=$ panic disorder, $\mathrm{SAD}=$ separation anxiety disorder, $\mathrm{MDD}=$ major depressive disorder, $\mathrm{GAD}=$ generalized anxiety disorder.

ADIS = Anxiety Disorders Interview Schedule, PANAS = Positive and Negative Affect Scale, BDI = Beck Depression Inventory, BAI $=$ Beck Anxiety Inventory, SCL-90 = Symptom Checklist-90, ADIS-C = Anxiety Disorders Interview Schedule-Child Version, RCMAS $=$ Revised Children's Manifest Anxiety Scale, CDI = Children's Depression Inventory, AFARS = Affect and Arousal Scale, PANAS-C = Positive and Negative Affect Scale for Children, PH-C, Physiological Hyperarousal Scale for Children, STAI = State-Trait Anxiety Inventory, ASI = Anxiety Sensitivity Inventory, $\mathrm{MPQ}=$ Multidimensional Personality Questionnaire, DIS = Diagnostic Interview Schedule. 
orders. Mineka and colleagues have proposed an integrative hierarchical model that includes tenets of the tripartite model and tenets of Barlow's hierarchical organization of anxiety disorders. Zinbarg and Barlow (1996) suggested that the emergence of one specific factor for anxiety disorders in models such as the tripartite model may be an artifact of lumping all the anxiety disorders together into a single group without recognizing important differences between the anxiety disorders. Mineka et al. (1998) hypothesized that each individual syndrome has its own unique component, such as anxious arousal that seems to be characteristic of panic disorder, in addition to a common component of NA and suggested that it is important for research studies to identify unique components of each anxiety disorder. The research reviewed in the present paper lends support to this hypothesis and elucidate the need for further research to identify unique components of anxiety disorders in youth.

There are several other limitations of research with the tripartite model specific to youth. One obvious problem in the literature is that far fewer studies have been conducted with youth samples compared to adult samples, despite the fact that anxiety and depression are prevalent in children and adolescents. Further examination of the tripartite model in youth populations is necessary because adult research suggests that the model is not applicable across anxiety disorders, and initial research with youth seems to be in accordance with these findings. Another problem with tripartite research in youth is that sole reliance on self-report measures, both traditional measures such as the RCMAS and CDI, and newer measures such as the PH-C and AFARS, is problematic due to the lack of discriminant validity among self-reports (Chorpita et al., 1998), as well as high associations among the tripartite constructs. The PH-C and AFARS show promise in more effective measurement of the tripartite factors, but more research is necessary. A related point of concern is that few studies rely on actual anxiety and depressive diagnoses derived from diagnostic interviews such as the ADIS-C (Chorpita et al., 1998). It is important to assess diagnostic status as well as symptom prevalence in order to determine if the tripartite model is applicable across severity levels of anxiety and depression. Another gap in the literature, as noted by Laurent and Ettelson (2001), is that research has yet to explore the connection between PH and objective physiological measures such as heart rate, which seems to be an important component of PH. Lastly, there is a paucity of studies with children and adolescents that have looked at the utility of the constructs NA, PA and PH with regard to specific anxiety disorders, and, thus, more research in youth is needed in order to determine whether it is appropriate to extrapolate the functioning of the tripartite constructs across anxiety disorders from adult research.

In addition to the limitations mentioned thus far, the research regarding the effects of gender and the tripartite model has been mixed. Some research has found no effect of gender with respect to the fit of the tripartite model (e.g., Huebner \& Dew, 1995; Ollendick et al., 2003), whereas other research has indicated that the tripartite model may not be generalizable across boys and girls (Jacques \& Mash, 2004; Joiner \& Blalock, 1995; Joiner, Blalock, \& Wagner, 1999; Lonigan et al., 1999). Research on gender differences in the tripartite model is far from conclusive, and thus it is necessary for future research to examine gender effects.

\subsection{Conclusions}

The present paper has outlined a number of findings. First, results from a number of investigations indicate that there is substantial overlap in anxiety and depressive symptoms and comorbid diagnoses in youth, yet these constructs can be adequately differentiated. Clark and Watson's tripartite model suggests that the overlap in anxiety and depression reflects a shared component of negative affect, and that low positive affect and physiological hyperarousal serve to differentiate anxiety and depression. Although initial research supported the utility of the tripartite model for explaining the association between anxiety and depression in adults and youth, in both clinical and nonclinical samples, a number of more recent investigations have called into question the applicability of the tripartite constructs. Evidence from a number of studies suggests that low PA is not only characteristic of depressive disorders and symptoms, as predicted by the tripartite model, but also is significantly associated with social phobia symptoms and diagnoses. Numerous investigations have found that NA seems to be more highly related to GAD compared to other anxiety disorders. Results furthermore have suggested that PH may be more significantly related to panic disorder versus other anxiety disorders. Overall, evidence thus indicates that the tripartite factors may not function similarly across all anxiety and depressive disorders. More research is necessary in children and adolescents in order to determine the functioning of tripartite constructs across anxiety disorders.

Based on this review, there are several steps necessary for future research in this area. First, research needs to identify the unique components of the tripartite model across youth samples with different anxiety disorders. Anxiety symptoms furthermore need to be assessed not only by self-report measures designed to measure either unspecified anxiety, such as the MASC, or specific anxiety symptoms, such as the SASC-R for social phobia symptoms, but also by diagnostic interview using the ADIS-C. Future research should not continue to rely on 
symptom measurement using the RCMAS and CDI, which have been shown to measure NA only when the total scales are used. Second, it will be important for the tripartite constructs to be assessed via newer selfreport measures specifically designed to assess these constructs, such as the AFARS and the PH-C. Third, future research should examine gender effects of the tripartite model because past research has been equivocal. Lastly, future research needs to examine the PH construct not only by self-report, but also by use of objective physiological measures, such as heart rate or blood pressure. Such objective measurements may provide a more multidimensional examination of the tripartite constructs.

\section{References}

Angold, A., \& Costello, J. E. (1993). Depressive comorbidity in children and adolescents: Empirical theoretical and methodological issues. American Journal of Psychiatry, 150, 1,779-1,791.

Axelson, D. A., \& Birmaher, B. (2001). Relation between anxiety and depressive disorders in childhood and adolescence. Depression and Anxiety, 14,67-78.

Beck, A., Epstein, N., Brown, G., \& Steer, R. (1988). An inventory for measuring clinical anxiety: Psychometric properties. Journal of Consulting \& Clinical Psychology, 56, 893-897.

Bittner, A., Goodwin, R. D., Wittchen, H., Beesdo, K., Hapfler, M., \& Leib, R. (2004). What characteristics of primary anxiety disorders predict subsequent major depressive disorder? Journal of Clinical Psychiatry, 65, 618-626.

Boyd, C. P., \& Gullone, E. (1997). An investigation of negative affectivity in Australian adolescents. Journal of Clinical Child Psychology, 26, 190-197.

Brady, E. U., \& Kendall, P. C. (1992). Comorbidity of anxiety and depression in children and adolescents. Psychological Bulletin, 111, 244-255.

Brown, T. A., Chorpita, B. F., \& Barlow, D. H. (1998). Structural relationships among dimensions of the DSM-IV anxiety and mood disorders and dimensions of negative affect positive affect and autonomic arousal. Journal of Abnormal Psychology, 2, 179-192.

Burns, D. D., \& Eidelson, R. J. (1998). Why are depression and anxiety correlated? A test of the tripartite model. Journal of Consulting and Clinical Psychology, 66, 461-473.

Chorpita, B. F., Albano, A. M., \& Barlow, D. H. (1998). The structure of negative emotions in a clinical sample of children and adolescents. Journal of Abnormal Psychology, 107,74-85.

Chorpita, B. F., \& Daleiden, E. L. (2002). Tripartite dimensions of emotion in a child clinical sample: Measurement strategies and implications for clinical utility. Journal of Consulting and Clinical Psychology, 70, 1,150-1,160.

Chorpita, B. F., Daleiden, E. L., Moffitt, C., Yim, L., \& Umemoto, L. A. (2000). Assessment of tripartite factors of emotion in children and adolescents I: Structural validity and normative data of an affect and arousal scale. Journal of Psychopathology and Behavioral Assessment, 22, 141-160.

Chorpita, B. F., Plummer, C. M., \& Moffitt, C. E. (2000). Relations of tripartite dimensions of emotion to childhood anxiety and mood disorders. Journal of Abnormal Child Psychology, 28, 299-310.

Clark, L. A., \& Watson, D. (1991). Tripartite model of anxiety and depression: Psychometric evidence and taxonomic implications. Journal of Abnormal Psychology, 100, 316-336.

Cole, D. A., Peeke, L. G., Martin, J. M., Truglio, R., \& Seroczynski, A. D. (1998). A longitudinal look at the relation between depression and anxiety in children and adolescents. Journal of Consulting and Clinical Psychology, 66, 451-460.

Cole, D. A., Truglio, R., \& Peeke, L. G. (1997). Relation between symptoms of anxiety and depression in children: A multitraitmultimethod-multigroup assessment. Journal of Consulting and Clinical Psychology, 65, 110-119.

Crook, K., Beaver, B., \& Bell, M. (1998). Anxiety and depression in children: A preliminary examination of the utility of the PANAS-C. Journal of Psychopathology and Behavioral Assessment, 20, 333-350.

Crowley, S. L., \& Emerson, E. N. (1996). Discriminant validity of self-reported anxiety and depression in children: Negative affectivity or independent constructs? Journal of Clinical Child Psychology, 25, 139-146.

Epkins, C. C., \& Meyers, A. W. (1994). Assessment of childhood depression, anxiety, and aggression: Convergent and discriminant validity of self-, parent-, teacher-, and peer-report measures. Journal of Personality Assessment, 62, 364-381.

Finch, A. J., Jr., Lipovsky, J. A., \& Casat, C. D. (1989). Anxiety and depression in children and adolescents: Negative affectivity or separate constructs? In:P. C. Kendall \& D. Watson (Eds.), Anxiety and Depression: Distinctive and Overlapping Features. San Diego, Calif.: Academic Press.

Huebner, E. S., \& Dew, T. (1995). Preliminary validation of the positive and negative affect schedule with adolescents. Journal of Psychoeducational Assessment, 13, 286-293.

Inderbitzen, H. M., \& Hope, D. A. (1995). Relationship among adolescent reports of social anxiety, anxiety, and depressive symptoms. Journal of Anxiety Disorders, 9, 385-396.

Jacques, H. A. K., \& Mash, E. J. (2004). A test of the tripartite model of anxiety and depression in elementary and high school boys and girls. Journal of Abnormal Child Psychology, 32,13-25.

Joiner, T. E., Jr. (1996). A confirmatory factor-analytic investigation of the tripartite model of depression and anxiety in college students. Cognitive Therapy and Research, 20, 521-539.

Joiner, T. E., Jr., \& Blalock, J. A. (1995). Gender differences in depression: The role of anxiety and generalized negative affect. Sex Roles, 33, 91-108. 
Joiner, T. E., Jr., Blalock, J. A., \& Wagner, K. D. (1999). Preliminary examination of sex differences in depressive symptoms among adolescent psychiatric patients: The role of anxious symptoms and generalized negative affect. Journal of Clinical Child Psychology, 28,211-219.

Joiner, T. E., Jr., Catanzaro, S. J., \& Laurent, J. (1996). Tripartite structure of positive and negative affect, depression, and anxiety in child and adolescent psychiatric inpatients. Journal of Abnormal Psychology, 105, 401-409.

Joiner, T. E., Jr., \& Lonigan, C. J. (2000). Tripartite model of depression and anxiety in youth psychiatric inpatients: Relations with diagnostic statues and future symptoms. Journal of Clinical Child Psychology, 29, 372-382.

Joiner, T. E., Jr., Steer, R. A., Beck, A. T., Schmidt, N. B., Rudd, M. D., \& Catanzaro, S. J. (1999). Physiological hyperarousal: Construct validity of a central aspect of the tripartite model of depression and anxiety. Journal of Abnormal Psychology, 108, 290-298.

King, N. J., Ollendick, T. H., \& Gullone, E. (1991). Negative affectivity in children and adolescents: Relations between anxiety and depression. Clinical Psychology Review, 11, 441-459.

Kovacs, M. (1992). Children's Depression Inventory Manual. North Tonawanda, NY: Multi-Health Systems.

Kovacs, M., Gastonis, C., Paulauskas, S. L., \& Richards, C. (1989). Depressive disorders in childhood: IV A longitudinal study of comorbidity with and risk for anxiety disorders. Archives of General Psychiatry, 46, 776-782.

LaGreca, A., \& Stone, W. (1993). Social anxiety scale for children revised: Factor structure and concurrent validity. Journal of Clinical Child Psychology, 22, 17-27.

Lambert, S. F., McCreary, B. T., Joiner, T. E., Schmidt, N. B., \& Ialongo, N. S. (2004). Structure of anxiety and depression in urban youth: An examination of the tripartite model. Journal of Consulting and Clinical Psychology, 72, 904-908.

Laurent, J., Catanzaro, S. J., \& Joiner, T. E., Jr. (2004). Development and preliminary validation of the physiological hyperarousal scale for children. Psychological Assessment, 16, 373-380.

Laurent, J., \& Ettelson, R. (2001). An examination of the tripartite model of anxiety and depression and its application to youth. Clinical Child and Family Psychology Review, 4, 209-230.

Lonigan, C. J., Carey, M. P., \& Finch, A. J., Jr. (1994). Anxiety and depression in children and adolescents: Negative affectivity and the utility of self-reports. Journal of Consulting and Clinical Psychology, 62, 1,000-1,008.

Lonigan, C. J., Hooe, E. S., David, C. F., \& Kistner, J. A. (1999). Positive and negative affectivity in children: Confirmatory factor analysis of a two-factor model and its relation to symptoms of anxiety and depression. Journal of Consulting and Clinical Psychology, 67, 374-386.

March, J. S., Parker, J. D. A., Sullivan, K., Stallings, P., \& Connors, C. K. (1997). The multidimensional scale for children (MASC): Factor structure, reliability, and validity. Journal of the American Academy of Child and Adolescent Psychiatry, 36, 554-565.

Merikangas, K. R., \& Avenevoli, S. (2002). Epidemiology of mood and anxiety disorders in children and adolescents. In: M. T. Tsuang \& M. Tohen (Eds.), Textbook in Psychiatric Epidemiology, 2nd edition (pp. 657-704). New York, N.Y.: Wiley-Liss.

Mineka, S., Watson, D. W., \& Clark, L. A. (1998). Psychopathology: Comorbidity of anxiety and unipolar mood disorders. Annual Review of Psychology, 49, 377-412.

Muris, P., Schmidt, H., Merckelbach, H., \& Schouten, E. (2001). The structure of negative emotions in adolescents. Journal of Abnormal Child Psychology, 29, 331-337.

Ollendick, T. H. (1983). Reliability and validity of the Revised Fear Survey Schedule for Children (FSSC-R). Behavior Research and Therapy, 21, 685-691.

Ollendick, T. H., Seligman, L. D., Goza, A. B., Byrd, D. A., \& Singh, K. (2003). Anxiety and depression in children and adolescents: A factor-analytic examination of the tripartite model. Journal of Child and Family Studies, 12, 157-170.

Ollendick, T. H., Shortt, A. L., \& Sander, J. B. (2005). Internalizing disorders of childhood and adolescence. In: J. E. Maddux \& B. A. Winstead (Eds.), Psychopathology: Foundations for a Contemporary Understanding (pp. 353-376). Mahwah, N.J.: Lawrence Erlbaum.

Ollendick, T. H., \& Yule, W. (1990). Depression in British and American children and its relation to anxiety and fear. Journal of Consulting and Clinical Psychology, 58, 126-129.

Ollendick, T. H., Yule, W., \& Ollier, K. (1991). Fears in British children and their relationship to manifest anxiety and depression. Journal of Child Psychology and Psychiatry and Allied Disciplines, 32, 321-331.

Orvaschel, H., Lewinsohn, P. M., \& Seeley, J. R. (1995). Continuity of psychopathology in a community sample of adolescents. Journal of the American Academy of Child and Adolescent Psychiatry, 23, 1,525-1,535.

Phillips, B. M., Lonigan, C. J., Driscoll, K., \& Hooe, E. S. (2002). Positive and negative affectivity in children: A multitraitmultimethod investigation. Journal of Clinical Child and Adolescent Psychology, 31, 465-479.

Reynolds, W. M. (1986). Reynolds Adolescent Depression Scale. Odessa, Fla.: Psychological Assessment Resources.

Reynolds, C. R., \& Richmond, B. O. (1978). What I think and feel: A revised measure of children's manifest anxiety. Journal of Abnormal Child Psychology, 6, 271-280.

Seligman, L. D., \& Ollendick, T. H. (1998). Comorbidity of anxiety and depression in children and adolescents: An integrative review. Clinical Child and Family Psychology Review, 1, 125-144.

Silverman, W. K., \& Albano, A. M. (1996). Anxiety Disorders Interview Schedule for DSM-IV: Child Version (manual). San Antonio: Psychological Corporation.

Spence, S. H. (1998). A measure of anxiety symptoms among children. Behaviour Research E Therapy, 36, 545-566.

Speilberger, C. D., Gorsuch, R. L., \& Lushene, R. E. (1970). Manual for the State-Trait Anxiety Inventory (self-evaluation questionnaire). Palo Alto, Calif.: Consulting Psychologists Press. 
Stavrakaki, C., Vargo, B., Boodoosingh, L., \& Roberts, N. (1987). The relationship between anxiety and depression in children: Rating scales and clinical variables. Canadian Journal of Psychiatry, 32, 433-439.

Stark, K. D., \& Laurent, J. (2001). Joint factor analysis of the children's depression inventory an the revised children's manifest anxiety scale. Journal of Clinical Child Psychology, 30, 552-567.

Strauss, C. C., Last, C. G., Hersen, M., \& Kazdin, A. E. (1988). Association between anxiety and depression in children and adolescents with anxiety disorders. Journal of Abnormal Child Psychology, 16, 57-68.

Tellegen, A., Watson, D., \& Clark, L. A. (1999). On the dimensional and hierarchical structure of affect. Psychological Science, 10, 297-303.

Turner, C. M., \& Barrett, P. M. (2003). Does age play a role in the structure of anxiety and depression in children and youths? An investigation of the tripartite model in three age cohorts. Journal of Consulting and Clinical Psychology, 71, 826-833.

Valentiner, D. P., Gutierrez, P. M., \& Blacker, D. (2002). Anxiety measures and their relationship to adolescent suicidal ideation and behavior. Journal of Anxiety Disorders, 16, 11-32.

Watson, D. (2005). Rethinking the mood and anxiety disorders: A quantitative hierarchical model for DSM-IV. Journal of Abnormal Psychology, 114, 522-536.

Watson, D., Clark, L. A., \& Carey, G. (1988). Positive and negative affectivity and their relation to anxiety and depressive disorders. Journal of Abnormal Psychology, 97, 346-353.

Watson, D., Clark, L. A., \& Tellegen, A. (1988). Development and validation of brief measures of positive and negative affect: The PANAS scales. Journal of Personality and Social Psychology, 54, 1,063-1,070.

Watson, D., Clark, L. A., Weber, K., Assenheimer, J. S., Strauss, M. E., \& McCormick, R. A. (1995). Testing a tripartite model: II Exploring symptom structures of anxiety and depression in student, adult, and patient samples. Journal of Abnormal Psychology, 104, 15-25.

Watson, D., Gamez, W., \& Simms, L. J. (2005). Basic dimensions of temperament and their relation to anxiety and depression: A symptom-based perspective. Journal of Research in Personality, 39, 46-66.

Watson, D., Weber, K., Assenheimer, J. S., Clark, L. A., Strauss, M. E., \& McCormick, R. A. (1995). Testing a tripartite model: I Evaluating the convergent and discriminant validity of anxiety and depression symptom scales. Journal of Abnormal Psychology, 104, 3-14.

Zinbarg, R. E., \& Barlow, D. H. (1996). Structure of anxiety and the anxiety disorders: A hierarchical model. Journal of Abnormal Psychology, 105, 181-193. 